* Events wholly or partially sponsored by IUGS, one of its Affiliated Associations or joint ventures -the International Geological Correlation Programme (IGCP) or the Inter-Union Commission on the Lithosphere (ICL).

- First announcement of meeting in EPISODES.

November 1984

MINERAL ECONOMICS OF THE WEST AFRICAN

BASEMENT (Workshop), Accra, Ghana. (CIFEG

103, rue de Lille, 75007 Paris, France)

November/December 1984

LAND EVALUATION FOR SOIL EROSION HAZARD ASSESSMENT (Meeting), Enschede, The Netherlands. (Dr. W.G. Sombroek, ISSS, International Soil Museum, 9 Duivendaal, POB 353,6700 A.J. Wageningen, The Netheriands)

November 5 - 8, 1984

GEOLOGICAL SOCIETY OF AMERICA (Annual Meeting), Reno, Nevada, U.S.A. (S.S. Beggs, Geological Society of America, P.O. Box 9140, 3300 Penrose Place, Boulder, CO 80301 , U.S.A.)

November 5 - 9, 1984

GEOPRESSURES AND HYDROCARBON OCCURRENCES (Symposium), Banff, Alberta, Canada. (Mrs. Pat Larlham, Faculty of Extension, The University of Alberta, Edmonton, Alberta, Canada $\mathrm{T} 6 \mathrm{G} 2 \mathrm{G} 4$ )

November $5-9,1984$

ARGENTINE GEOLOGICAL CONGRESS (9th), Bariloche, Argentina. Field trips. Languages: Spanish, English, and French. (IX Congreso Geologico Argentino, Maipu 645 Piso 1, 1006 Buenos Aires, Argentina)

November $6-7,1984$

NORTH ATLANTIC PALAEOOCEANOGRAPHY (Marine Studies Group Meeting), London, U.K. (N. Shackleton, Department of Quaternary Research, Godwin Laboratory, Free School Lane, Cambridge CB2 3RS, U.K.)

November $12-17,1984$

WATER FOR SOUTH AFRICA (Exposition and Conference), Johannesburg, South Africa. Sponsored by National Water Well Association (U.S.) and Borehole water Association (S.A.). (Pat Alcorn, NWWA, 500 W. Wilson Bridge Road, Worthington, Ohio 43085 , U.S.A.)

November $12-30,1984$

RURAL HYDROGEOLOGY AND HYDRAULICS IN FISSURED BASEMENT ZONES (Workshop), Roorkee, India. (Prof. B.B.S. Singhal, Department of Earth Sciences, University of Roorkee, Roorkee 247667 , India)

November $13-15,1984$

OPHIOLITES TROUGH TIME (Conference), Nancy, France. (J. Desmons, Université de Nancy I, Laboratorie de Pétrologie, B.P. 239, F-54506 Vandoeuvre-lès-Nancy Cedex, France)

November $15-17,1984$

* MINERAL POLICY FOR SMALl-SCALE MiNING (Workshop), New Delhi, India. Cosponsored by AGID in conjunction with World Mining Congress. (Co-ordinator, Regional Mineral Resources Development Centre, P.O. Box 19, Bandung, Indonesia)

November $19-23,1984$

- CENTRAL AMERICA (6th Geological Congress and lst Mining Symposium), Managua, Nicaragua. Languages: Spanish and English. (Mrs. Gilda Amador, Apartado Postal 195, Managua, Nicaragua)

November $19-23,1984$

WORLD MINING (12th Congress), New Delhi, India. Corganizing Committe, Institute of Engineers, 8 Gokhale Road, Calcutta 700020 , India)

November $27-30,1984$

- GEOBOTANY AND BIOGEOCHEMISTRY IN EXPLORATION FOR GROUND WATER AND MINERAL RESOURCES (International Seminar),
Tirupati, India. (Dr. E.A.V. Prasad, Department of Geology, Sri Venkateswara University, Tirupati 517502 (A.P.), India)

December 1984

* CLIMAT - MILIEU - HOMME (Colloquium), Dakar, Senegal. Cosponsored in part by INQUA and Unesco. (Dr. E.S. Diop, University of Dakar, Department of Geography, Dakar Fann, Senegal)

Decenber $2-5,1984$ FUTURE PETROLEUM PROVINCES OF THE WORLD (AAPG W.E. Pratt Memorial Conference), Phoenix, Arizona, U.S.A. (AAPG, P.O. Box 979, Tulsa, OK 74101, U.S.A.)

December $2-6,1984$

SOCIETY OF EXPLORATION GEOPHYSICISTS (54th Annual Meeting), Atlanta, Georgia, U.S.A. (J. Hyden, SEG, Box 3098, Tulsa, OK 74101 , U.S.A.)

\section{December $3-7,1984$}

AMERICAN GEOPHYSICAL UNION (F all Neeting), San Francisco, California, U.S.A. (AGU Meetings, 2000 Florida Avenue N.W., Washington, DC 20009 , U.S.A.)

December 3 - 14, 1984

- GEOTECHNICAL ASPECTS OF MASS AND MATERIAL TRANSPORTATION (Symposium \& Specialty Sessions), Bangkok, Thailand. (Prof. A.S. Balasubramaniam, Division of Geotechnical \& Transportation Engineering, Asian Institute of Technology, G.P.O. Box 2754, Bangkok 10501, Thailand)

December $9-13,1984$

- JORNADA DE GEOFISICA of Central America and the Caribbean (Ist Geophysical Meeting), Panama City, Panama. (Instituto de Geociencias, Universidad de Panama, Panama)

December $10-14,1984$

- NEW ZEALAND GEOLOGICAL SOCIETY (Annual Conference), Wellington, New Zealand. (New Zealand Geological Society, Department of Geology, Victoria University of Wellington, Private Bag, Wellington, New Zealand)

\section{5}

January 1985 ACID-SULPHATE SOILS (Meeting), Dakar, Senegal. (Dr. W.G. Sombroek, ISSS, International Soil Museum, 9 Duivendaal, POB 353, 6700 A.J. Wageningen, The Netherlands)

January $7-10,1985$

^ HYDROGEOLOGY OF ROCKS OF LOW PERMEABILITY (17th International Congress of IAH), Tucson, Arizona, U.S.A. (Eugene S. Simpson, Department of Hydrology and Water Resources, College of Earth Sciences, The University of Arizona, Tueson, AZ 85721 , U.S.A.)

February 1985

- RECENT CRUSTAL MOVEMENT (International Symposium), Maracaibo, Venezuela. (Heinz Henneberg, International Association of Geodesy, Apartado 6, Maracaibo, Venezuela)

February 1985

INTERGOVERNMENTAL OCEANOGRAPHIC COMMISSION ASSEMBLY (13th Session), Paris, France. (Unesco, 7, place de Fontenoy, 75700 Paris, France)

February 1 - 10, 1985

* PAN-AFRICAN CRUSTAL EVOLUTION IN ARABIA AND NORTHEAST AFRICA (2nd IGCP 164 Symposium), Jeddah, Saudi Arabia. Field excursions. (Dr. Ahmed Al-Shanti, P.O. Box 1744, Jeddah 21441 , Saudi Arabia)
February 11 - 14, 1985

GEOMECHANICS IN TROPICAL LATERITE AND SAPROLITIC SOILS (lst International Conference), Sao Paulo, Brazil. (Dr. W.C. Hachich, Secretary ISTS-BMS, C.P. 7141, 01000 Sao Paulo, $\mathrm{SP}$, Brazil)

February $11-14,1985$

ASIAN MINING 85 (2nd Conference), Manila, Philippines. (Meeting Secretary, The Institution of Mining and Metallurgy, 44 Portland Place, London WiN 4BR, U.K.)

February $20-21,1985$

- EXTRAORDINARY FOSSIL BIOTAS: Ecological and Evolutionary Significance (International Discussion Meeting), London, U.K. (S. Conway Morris, Department of Earth Sciences, Dowing Street, Cambridge CB2 $3 \mathrm{EQ}$, U.K.)

February $24-28,1985$

AMERICAN INSTITUTE OF MINING, METALLURGICAL AND PETROLEUM ENGINEERS (Annual Meeting), New York, U.S.A. (AIME, 345 East 47th Street, New York, NY 10017 , U.S.A.)

February $24-28,1985$

SALTS AND BRINES 85 (Meeting), New York, U.S.A. (William C. Larson, Twin Cities Research Center, Bureau of Mines, 5629 Minnehaha Avenue S, Minneapolis, MN 55417, U.S.A.)

February 25, 1985

PAUL F. KERR Memorial Symposium, New York, U.S.A. (Otto C. Kopp, Department of Geological Sciences, University of Tennessee, Knoxville, TN 37996-1410, U.S.A.)

February 25 - March 1, 1985

TROPICAL PEAT RESOURCES: PROSPECTS AND POTENTIAL (Symposium), Kingston, Jamaica. (Dr. Barry Wade, IPS Symposium, Petroleum Corporation of Jamaica, P.O. Box 579, Kingston 10, Jamaica, W.1.)

February 27 - March 2, 1985

$\star$ GEOLOGY OF THE OCEANS (75th Annual Meeting of the Geologische Vereinigung), Kiel, West Germany. Sponsored by IUGS Commission for Marine Geology and Working Group 7 of JI.C. Languages: English and German. (M. Sarnthein, Ceologisch-Palaeontologisches Institut, Universität, Olshausenstrasse, D-2300 Kiel, Federal Republic of Germany)

March $10-15,1985$

COAL (International Conference), Tel Aviv, Israel. (Kenes, P.O. Box 50006, Tel Aviv 61 500, Israel)

March $11-15,1985$

SE ASIAN GEOTECHNICAL CONFERENCE (8th), Kuala Lumpur, Malaysia. (The Hon. Secretary, 8 th SEAGC, The Institution of Engineers, Malaysia, P.O. Box 223, Petaling Jaya, Selangor, Malaysia)

March $11-15,1985$

TUNNELLING 85 (4th International Symposium), Brighton, England. (Tunnelling 85, The Secretary, Institution of Mining and Metallurgy, 44 Portland Place, London WIN 4BR, U.K.)

March $11-15,1985$

- lunar and planetary science (Conference), Houston, Texas, U.S.A. (Pamela Jones, Lunar \& Planetary Institute, 3303 NASA Road 1, Houston, TX 77058 , U.S.A.)

March $20-21,1985$

- PETROLEUM AND MINERALS REVIEW (Conference), Canberra, Australia. (E.E. Young, BMR, Department of Resources and Energy, Box 378 , Canberra, ACT 2601, Australia). 
March 24-27, 1985

- AMERICAN ASSOCIATION OF PETROLEUM GEOLOGISTS (Annual Convention), New Orleans, U.S.A. (George Severson, The Louisiana Land and Exploration Co., P.O. Box 60350, New Orleans, LA 70160 , U.S.A.)

March $24-30,1985$

^GEOCHEMICAL EXPLORATION IN TROPICAL RAIN FORESTS (International Workshop), Manaus, Brazil. Sponsored by AEG and AGID. (Richard W. Lewis, Jr., Geoquimica Ltda., Rau Aguaraiba, 86, Bonsucesso, Rio de Janeiro, RJ-Brazil)

March $25-26,1984$

- EXTRACTIVE INDUSTRY GEOLOGY (4th Conference), Nottingham, U.K. (Conference Department, British Geological Survey, Exhibition Road, London SW 7 2DE, U.K.)

March 28-29, 1985

- MAJOR CRUSTAL LINEAMENTS AND THEIR INFLUENCE ON THE GEOLOGICAL HISTORY OF THE CONTINENTAL LITHOSPHERE (Royal Society Discussion Meeting), London, U.K. (J.V. Watson, Department of Geology, Imperial College of Science and Technology, Prince Consort Road, London SW7 2BP, U.K.)

April $1-4,1985$

EUROPEAN UNION OF GEOSCIENCES (Biennial Conference), Strasbourg, France. (Organizing Committee, Department of Earth Sciences, University of Cambridge, Downing Street, Cambridge CB2 3EQ, U.K.)

April $1-5,1985$

NUMERICAL METHODS IN GEOMECHANICS (5th International Conference), Nagoya, Japan. (Prof. T. Kawamoto, Department of Civil \& Geotechnical Engineering, Nagoya University, Chikusa, Nagoya 464, Japan)

April 9-12, 1985

EVOLUTION OF THE EUROPEAN LITHOSPHERE (MEGS 4: Meeting of European Geological Societies), Edinburgh, U.K. (Dr. S.K. Monro, Institute of Geological Sciences, Murchison House, West Mains Road, Edinburgh EH9 3LA, Scotland, U.K.)

April $10-12,1985$

MACRO-MESO-MICRO (Conference on structural geology), Utrecht, The Netherlands. (Vakgroep Structurele Geologie, Instituut voor aardwetenschappen, Budapestlaan 4, Ryksuniversiteit, Utrecht, The Netherlands)

April $14-17,1985$

PROSPECTING IN AREAS OF DESERT TERRAIN (Conference), Rabat, Moroceo. (Conference Office, IMM, 44 Portland Place, London WIN $4 \mathrm{BR}$, U.K.)

April $14-19,1985$

- GEOMORPHIC AND STRATIGRAPHIC INDICATORS OF NEOGENE-QUATERNARY CLIMATIC CHANGE IN ARID AND SEMI-ARID ENVIRONMENTS (GSA Penrose Conference), Lake Havasu City, Arizona, U.S.A. (John Dohrenwend, U.S. Geological Survey, MS 941, 345 Middlefield Road, Menlo Park, CA 94025, U.S.A.)

April 15-16, 1985

DEFORMATION MECHANISMS IN SEDIMENTS AND SEDIMENTARY ROCKS (Geological Society of London Conference), London, U.K. (Dr. M.E. Jones, Department of Geology, Kings College London, Strand, London WC2, U.K.)

April 15 - 17, 1985

- INTERNATIONAL ASSOCIATION OF SEDIMENTOLOGISTS (6th Regional Metting), Lleida, Spain. (Joan Rosell, Dpt. Geologia, Universitat Autonoma, Bellaterra, Barcelona, Spain)

April $15-19,1985$

ANALYTICAL CHEMISTRY IN THE EXPLORATION, MINING AND PROCESSING OF MATERIALS (2nd International Symposium),
Pretoria, South Africa. (Symposium Secretariat, S.328, CSIR, P.O. Box 395, Pretoria 0001, South Africa)

April $15-19,1985$

NON-METALLIC MINERALS (World Congress), Belgrade, Yugoslavia. Languages: English, Russian, Serbo-Croatian. (Svetski Kongres O Nemetalicnim Mineralnim, Sirovinama, Sava Centar, 11070 Beograd, Yugoslavia)

April $16-19,1985$

- MINING TECHNOLOGY: MINING IN SOFT GROUND (International Forum), Nottingham, U.K. (J. Snedegar, 223 Transportation Research Building, University of Kentucky, Lexington, KY 40506, U.S.A.)

April 18 - 20, 1985

CONTINENTAL EXTENSIONAL TECTONICS (Meeting), Durham, U.K. (J.F. Dewey, Department of Geological Sciences, University of Durham, Science Laboratories, South Road, Durham DHI 3LE, U.K.)

April $23-26,1985$

GROUNDWATER MANAGEMENT (Conference), Berlin. (The Secretary-General, International Water Supply Association, 1 Queen Anne's Gate, London SW1H 9BT, U.K.)

April 28 - May 1, 1985

$\star$ GEOCHEMICAL EXPLORATION (11th International AEG Symposium), Toronto, Canada. (Dr. W.B. Coker, Kidd Creek Mines Ltd., 357 Bay St., Ste. 300, Toronto, Ontario, Canada M5H 1T7)

April 30 - May 3, 1985

- PACIFIC COAL (lst Conference and Exhibition), Brisbane, Australia. (J. Prentice, Conventions Queensland, 30 McCaul Street, Taringa 4068, Queensland, Australia)

May $1-2,1985$

OCEAN FRACTURE ZONES (Meeting), London, U.K. (R.S. White, c/o Carolyn Symonds, Geological Society of London, Burlington House, Piccadilly, London W l, U.K.)

May $6-17,1985$

^ NEOGENE PHOSPHORITES OF THE SE UNITED STATES (International field workshop and sem:nar, IGCP 156), Greenville, N.C. to Tallahassee, Florida. (W.C. Burnett, Dept. of Oceanography, Florida State University, Tallahassee, FL 32306 , U.S.A.)

May $13-17,1985$

TUNGSTEN (3rd International Symposium), Madrid. (Mr. M.R.P. Maby, Secretary, Primary Tungsten Association, 280 Earls Court Road, London SW5 9AS, U.K.)

May $15-17,1985$

TURBIDITE-HOSTED GOLD DEPOSITS (International Symposium), Fredericton, New Brunswick, Canada. (Simon J. Haynes, Nova Scotia Department of Mines and Energy, P.O. Box 1087, 1690 Hollis Street, Halifax, Nova Scotia, Canada B3J 2X1)

May $15-18,1985$

GEOLOGICAL, MINERALOGICAL ASSOCIATIONS OF CANADA (Joint Annual Meeting), Fredericton, New Brunswick, Canada. (Dr. W. Van de Poll, Department of Geology, University of New Brunswick, Fredericton, New Brunswick, Canada E3B 5A3)

May $20-25,1985$

* METALLOGENESIS OF THE PRECAMBRIAN IN EUROPE (International Conference), Tabor, Central Bohemia, Czechoslovakia. Sponsored by IGCP-91. (International Conference on Metallogenesis of the Precambrian in Europe, Attention Dr. Gabriel, Malostranské nam. 19, 118 21 Praha, Czechoslovakia)

May $26-31,1985$

$\star$ EUROPEAN ASSOCIATION OF SCIENCE EDITORS (2nd Conference), Holmenkollen, Norway.
(Nancy Morris, EASE Secretariat, P.O. Box 33, Farnham, Surrey GU $103 \mathrm{JX}$, U.K.)

May $27-31,1985$

AMERICAN GEOPHYSICAL UNION (Spring Meeting), Baltimore, Maryland, U.S.A. (Meetings, AGU, 2000 Florida Avenue, NW, Washington, DC 20009 , U.S.A.)

May 27 - June 1, 1985

- CORAL REEF CONGRESS: Reef and Man (5th International), Papeete, Tahiti. Sponsored by IGCP 200, INQUA, ICL and IGU. (The Organizing Committee, Coral Reef Congress, Sea-level Meeting, B.P. 562, Papeete, Tahiti, French Polynesia)

May 27 - June 2, 1985

- TERRANES IN THE CIRCUM-ATLANTIC PALEOZOIC OCEANS (Penrose Conference), Liscombe Lodge, Nova Scotia, Canada. (J.D. Keppie, N.S. Depart. Mines and Energy, Box 1087 , Halifax, N.S. B3J 2X1, Canada)

June 1985

- SOIL DYNAMICS AND EARTHQUAKE ENGINEERING (2nd International Conference), on board the liner Queen Elizabeth 2. (Dr. C.A. Brebbia, Computational Mechanies Centre, Ashurst Lodge, Ashurst, Southamption, s04 $2 \mathrm{AA}$, U.K.)

June 2-9, 1985

INTERNATIONAL MINERAL PROCESSING CONGRESS (15th), Cannes, France. Languages French, English, German and Russian. (International Mineral Processing Congress Secretary, BRGM SGN/Mineralurgie, B.P. 6009-45060 Orléans Cedex, France)

June $4-7,1985$

EUROPEAN ASSOCIATION OF EXPLORATION GEOPHYSICISTS (47th Meeting), Budapest, Hungary. (E, van der Gaag, European Association of Exploration Geophysicists, P.O. Box 162, NL-2501 AN The Hague, The Netherlands)

June $4-7,1985$

* MAFIC DYKE SWARMS (International Conference), Toronto, Ontario, Canada. Sponsored by IUGS, ILP, and GSC. (Dr. H.C. Halls, Erindale Campus, University of Toronto, Mississauga, Ontario, Canada L5L 1 C6)

June $9-15,1985$

WATER RESOURCES (5th World Congress) Brussels, Belgium. (Dr. L.W. Debacker, c/o Brussels International Conference Centre, Parc des Expositions, Place de Belgique, B-1020 Brussels, Belgium)

June $10-14,1985$

MINING '85 (International Conference), Birmingham, U.K. (Institution of Mining Engineers, Danum House, South Parade, Doncaster DNl 2DY, U.K.)

June $16-21,1985$

SEISMICITY AND SEISMIC RISK (3rd International Symposium), Liblice, Czechoslovakia. (Dr. Z. Schenkova, Geophysical Institute, Bocni II, 14131 Prague 4, Czechoslovakia)

June $18-19,1985$

- MARINE MINERALS IN EXCLUSIVE ECONOMIC ZONES (Meeting), London, U.K. (S.A. Moorby, Geology Department Imperial College, London SW 7 2BP, U.K.)

July 7 - 12, 1985

AUSTRALASIAN INSTITUTE OF MINING AND METALLURGY (Annual Conference), Queensland, Australia. (AuslMM, P.O. Box 310, Carlton South, Victoria 3053, Australia)

July $7-19,1985$

KARST WATER RESOURCES (International Symposium), Antalya/Ankara, Turkey. Sponsored by IAHS. (Prof. G. Gunay, Hydrogeological Engineering Department, Hacettepe University, Beytepe, Ankara, Turkey) 


\section{Coming Events}

July $8-10,1985$

- HYDROLOGY (International Symposium), Fort Collins, Colorado, U.S.A. (H.W. Shen, Department of Civil Engineering, Hydrology and Water Resources Program, Foothills Campus, Colorado State University, Fort Collins, CO 80523 , U.S.A.)

July $10-12,1985$

- geOlogical SOCIETY OF TRINIDAD AND TOBAGO (lst Conference) Port-of-Spain, Trinidad, West Indies. Language: English. (Mr. Winston Ali, Programme Chairman, Geological Conference of the G.S.T.T., c/o Geological Department, Trinmar Ltd., Point Fortin, Trinidad, West Indies)

July $14-30,1985$

* MAGMATIC SUlfides IN MAFIC ROCK (IGCP 161 - Field Conference 4), Ketchikan, Alaska to Duluth, Minnesota, U.S.A. (Gerald K. Czamanske, M.S. 984, U.S. Geological Survey, Menlo Park, CA 94025, U.S.A.)

July $16-23,1985$

- CEPHAlOPODS: PRESENT AND PAST (2nd International Symposium), Tübingen, West Germany. (Prof. Jürgen Kullman, Geol. -Paläont, Institut, Sigwartstrasse 10, D-7400 Tübingen, Federal Republic of Germany)

July 27 - August 7,1985

PLATINUM (4th International Symposium), Duluth, Minnesota, U.S.A. to Sudbury, Ontario, Canada. (A.J. Naldrett, Department of Geology, University of Toronto, Toronto, Ontario, Canada $\mathrm{M} 5 \mathrm{~S} 1 \mathrm{Al})$

July 28 - August 2,1985

^ ClAY (8th International Conference), Denver, Colorado, U.S.A. Sponsored by AIPEA. (Dr. A.J. Herbillon, Groupe de Physico-Chimie Minérale et de Catalyse, Univ. Catholique de Louvain, Place Croix du Sud 1, B-1348 Louvain-la-Neuve, Belgium)

July 29 - August 1,1985

OSTRACODA (9th International symposium) Shizuoka, Japan. (Dr. Tetsuro Hanai, Institute of Geosciences, University of Shizuoka, Shizuoka, 422, Japan)

July 29 - August 9,1985

TSUNAMI 85 (International Symposium), Victoria, British Columbia, Canada. (Tsunami 85 , P.O. Box 2267, Sidney, B.C., Canada V8L 3S8)

August $5-16,1985$

IAMAP/IAPSO (Joint Assembly), Honolulu, Hawaii, U.S.A. (Joint Assembly, American Geophysical Union, 2000 Florida Avenue NW, Washington, DC 20009 , U.S.A.)

August $5-17,1985$

MAGNETIC ANOMALIES OVER MARGINS OF CONTINENTS AND PLATES (IAGA Symposium), Prague, Czechoslovakia. (Mr. Vaclav Bucha, Geophysical Institute, Acad. Sco., Bocni 11, 14131 Prague 4, Czechoslovakia)

August $6-10,1985$

SCIENCE AND TECHNOLOGY EDUCATION AND THE QUALTTY OF LIFE (International Conference), Bangalore, India. Sponsored by ICSU/CTS (J. Lewis, Physies Department, Malvern College, Malvern, Worcs., U.K.)

August $6-10,1985$

GROUND FREEZING (4th International Symposium), Sapporo, Japan. (ISGF85, Institute of Low Temperature Science, Hokkaido University, Sapporo 060, Japan)

August $11-15,1985$

INTERNATIONAL SOCIETY FOR SOIL MECHANICS AND FOUNDATION ENGINEERING (11th International Conference), San Francisco, California, U.S.A. (K. Hyland, American Society of Civil Engineers, 345 East 47 th Street, New York, NY 10017 , U.S.A.)

A ugust $12-14,1985$

- HYDROTHERMAL REACTIONS (2nd International Symposium), University Park, Penr. U.S.A.
(Symposium) on Hydrothermal Reactions, The Pennsylvania State University, Keller Conference Center, University Park, PA 16802, U.S.A.)

August $12-16,1985$

OCCURRENCE, PROPERTIES, AND UTILIZATION OF NATURAL ZEOLITES (Internationa Conference), Budapest, Hungary. (Dr. J. Engelhardt, Central Research Institute for Chemistry, Hungarian Academy of Sciences, H-1525 Budapest, P.O. Box 17, Hungary)

August $19-23,1985$

^ GONDWANA (6th symposium), Columbus, Ohio, U.S.A. Sponsored by IUGS and Geological Society of America. (D. Elliott, Institute of Polar Studies, Ohio State University, 103 Mendenhall, 125 South Oval Mall, Columbus, OH 43210 , U.S.A.)

August $19-24,1985$

CHILEAN GEOLOGICAL CONGRESS (4th) Antofagasta, Chile. (4th Chilean Geological Congress, Department of Geosciences, Universidad del Norte, Casilla Box 1280, Antofagasta, Chile)

August $19-30,1985$

IASPEI (23rd General Assembly), Tokyo, Japan. (Prof. R. Sato, c/o Inter Group Corp., Akasaka Yamakatsu Building, 8-5-32 Akasaka, Minato-ku, Tokyo 107, Japan)

August $21-23,1985$

PALEOENVIRONMENTAL RECONSTRUCTION OF THE LATE WISCONSIN DEGLACIATION AND THE HOLOCENE, Lethbridge, Alberta, Canada, (Dr. René W. Barendregt, Department of Geography, The University of Lethbridge, 4401 University Drive, Lethbridge, Alberta, Canada TIK 3M4)

August $23-25,1985$

- HIGH TEMPERATURE GEOTHERMAL DRILLING (Workshop), Kailua-Kona, Hawaii, U.S.A. (Geothermal Resources Council, P.O. Box 1350 , Davis, CA 95617-1350, U.S.A.)

A ugust 24 - September 2, 1985

* GRAPTOLITES (3rd International Conference, Graptolite Working Group IPA), Helsingor, Denmark. (Dr. M. Bjerreskov, Institute of Historical Geology and Palaeontology, University of Copenhagen, Oster Voldgade 10, DK-1350, Copenhagen $\mathrm{K}$, Denmark)

August $26-29,1985$

GLACIER MAPPING AND SURVEYING (Symposium), Reykjavik, Iceland. (Mrs. H. Richardson, Secretary General, International Glaciological Society, Lensfield Road, Cambridge CB2 IER, U.K.)

A ugust $26-30,1985$

GEOTHERMAL ENERGY (International Symposium), Hawaii, U.S.A. (Geothermal Resources Council, P.O. Box 1350, Davis, CA 9561 7, U.S.A.)

August $27-30,1985$

- INTERNATIONAL MINING HISTORY (Conference), Melbourne, Australia. (P. Richardson, Economic History Department, University of Melbourne, Parkville, Victoria 3052, Australia)

August 31 - September 1, 1985

- FRACTURES IN GEOTHERMAL RESERVOIRS OR GEOTHERMAL EXPLORATION (Workshop) Kailua-Kona, Hawaii, U.S.A. (Geothermal Resources Council, P.O. Box 1350, Davis, CA 95617-1350, U.S.A.)

September 1985

ASSESSMENT OF THE ROLE OF $\mathrm{CO}_{2}$ ON CLIMATE VARIATIONS AND THEIR IMPACTS (Meeting), Austria. (Prof. L. Ernster, 51 boulevard de Montmorency, F-75016 Paris, France)

September 1985

VARIATIONAL METHODS IN GEOSCIENCES (International Symposium), Norman, Oklahoma, U.S.A. (CIMMS, Attn: Y.K. Sasaki, The University of Oklahoma, 815 Jenkins, Norman, Oklahoma 73019 , U.S.A.)
September 1985

DEEP INTERNAL PROCESSES AND CONTINENTAL RIFTING (International Symposium), Chengdu, P.R. China. Co-sponsored by ICL and Chinese Lithospheric Committee. Languages: Chinese and English. (Claude Froidevaux, Université Paris-Sud, Lab. Géophysique-Bât. 510 , 91405 Orsay, France; Tan Tjong Kie, Institute of Geophysics, Academia Sinica, Pekin, People's Republic of China)

September $2-4,1985$

ROLE OF ROCK MECHANICS IN MINING (International Symposium), Mexico City, Mexico. Sociedad Mexicana de Mecanica de Rocas AC, Camino a Santa Teresa 187, Villa Olimpica, MEX14020 Mexico DF, Mexico)

September $2-7,1985$

- FORELAND BASINS (Research Symposium), Fribourg, Switzerland. Sponsored by IAS. (Dr. P. Homewood, Institut de Géologie, Université de Fribourg, Pérolles, CH-1700 Fribourg, Switzerland)

September $3-5,1985$ EROSION DEBRIS FLOW AND DISASTER PREVENTION (International Symposium), Tsukuba, Japan. (Dr. S. Kobaski, ISEDD 1985, Department of Forestry, Kyoto University, Kyoto 606, Japan)

September $5-10,1985$

CARPATHO-BALKAN GEOLOGICAL ASSOCIATION (13th Congress), Krakow, Poland. (Oddzial Karpacki Instytutu Geologiczhego, ul. Skrzatow 1, 31-560 Krakow, Poland)

September 8 - 13, 1985

* HYDROGEOLOGY IN THE SERVICE OF MAN (18th IAH Congress - International Symposium), Cambridge, U.K. (J. Day, Hydrogeology Unit, Maclean Building, Crowmarsh Gifford, Wallingford, $0 \times 108 \mathrm{BB}$, U.K.)

September $9-12,1985$

- EXTRACTION METALLURGY (International Symposium), London, U.K. (Conference Office, The Institution of Mining and Metallurgy, 44 Portland Place, London WIN 4BR, U.K.)

September $9-13,1985$

FOSSIL AND LIVING BRACHIOPODS (Meeting), Brest, France. (Congrès Brachiopodes, Univ. Bretagne Occidentale, Laboratoire du Paléozoique - 6, av. Le Gorgen, 29283 Brest Cedex, France)

September $15-20,1985$

BASEMENT TECTONICS (6th International Conference), Santa Fe, New Mexico, U.S.A. (Dr. M. James Aldrich, Mail Stop D 461, P.O. Box 1663 Los A lamos National Laboratory, Los Alamos, NM 87545, U.S.A.)

September $15-21,1985$

GEOMORPHOLOGY, RESOURCES, ENVIRONMENT AND THE DEVELOPING WORLD (International Conference), Manchester, U.K. (Prof. Ian Douglas, School of Geography, University of Manchester, Manchester Ml3 9PL U.K.)

September $15-22,1985$

REGIONAL COMMITTEE ON MEDITERRANEAN NEOGENE STRATIGRAPHY (8th Congress), Budapest, Hungary. Language: English. (Organizing Committee of the 8th Congress of the RCMNS, Hungarian Geological Survey, Népstadion ut 14. H-l 442 Budapest, P.O.B. (Pf.) 106 Hungary)

September $16-21,1985$

GEOPHYSICAL AND PETROLOGICAL CONSTRAINTS TO MAGMA GENERATION including Symposia on Potassic Volcanism and Etna Volcano (IAVCEI Scientific Assembly), Catania, Italy. (G. Frazetta, Istituto Internazionale di Vulcanologia, Viale Regina Margherita 6, 95123 Catania, Italy)

September $17-21,1985$

- INTERNATIONAL MINE WATER ASSOCIATION (2nd International Congress), Granada, Spain. (R.F. Rubio, School of Mines, Technical University, Rios Rosas 21, Madrid 3, Spain) 


\section{Coming Events ...}

September $19-21,1985$

GEOLOGICAL SOCIETIES OF THE BRITISH ISLES (6th Meeting), Birmingham, U.K. (Prof. P.A. Garrett, Department of Geological Sciences, The University of Birmingham, P.O. Box 363 , Edgbaston, Birmingham Bl 5 2TT, U.K.)

September $22-26,1985$

HIGH HEAT PRODUCTION, GRANITES, HYDROTHERMAL CIRCULATION AND ORE GENESIS (Conference), St. Austell, Cornwall, U.K. (IMM, 44 Portland Place, London W1N 4BR, U.K.)

September $22-28,1985$

CHEMRAWN IV: CHEMISTRY AND RESOURCES OF THE GLOBAL OCEAN (Meeting), Woods Hole, Mass., U.S.A. (Prof. G. Ourisson, Centre de Neurochimie, Université Louis Pasteur, 5 rue Blaise Pascal, F-67084 Strasbourg, France)

October $6-10,1985$

SOCIETY OF EXPLORATION GEOPHYSICISTS (55th Annual Meeting), Washington, D.C., U.S.A, (Convention Assistant, Society of Exploration Geophyseists, P.O. Box 3098, Tulse, OK 74101, U.S.A.)

October $6-10,1985$

- GEOLOGY OF VEnEZuela (6th Congress), Caracas, Venezuela. (Secretaria General, VI Congreso Geologico Venezolano, Sociedad Venezolana de Geologos, Apartado 2006, Caracas 1010 - A Venezuela)

October $9-10,1985$

* MANAGEMENT OF HAZARDous Chemical WASTE SITES (IAEG International Symposium), Winston Salem, N.C., U.S.A. (International Symposium, c/o Ebaseo Services Ine., 2211 Meadowview Road, Greensboro, NC 27401, U.S.A.)

October $9-12,1985$

* LATIN AMERICA (6th Geological Congress), Bogota, Colombia. Languages: Spanish, English, French, and Portuguese. (VI Latin American Geological Congress, INGEOMINAS-AGID, Diagonal 53 No. 34-53, P.O. Box 4865, Bogota, D.E., Colombia)

October $10-12,1985$

HEAT AND DETACHMENT IN CRUSTAL EXTENSION ON CONTINENTS AND PLANETS (Topical Conference), Sedona, Arizona, U.S.A. (Pam Jones, LPl, I Nasa Road, Houston, TX 77058, U.S.A.)

October $14-17,1985$

*• LATERITE (International Seminar), Tokyo, Japan. IGCP. (Dr. Y. Ogura, International Seminar on Laterite, $\mathrm{c} / \mathrm{o}$ The Mining and Metallurgical Institute of Japa, 5-4 Ginza, 8-Chrome, Chuo-ku, Tokyo 104, Japan)

Oetober $14-18,1985$

COAL RESEARCH (7th International Conference), Pretoria, South Afriea. (W.G. Jensen, International Committee for Coal Research, Bte 11, B-1150 Bruxelles, Belgium)

October $14-18,1985$

MATHEMATICAL METHODS IN GEOLOGY (International Symposium), Pribram, Czechoslovakia. (Sekretariat symposia, Hornicka Pribram ve vede a Technice, post. schranka 41, Pribram 26102 , Czechoslovakia)

October $20-23,1985$

- UNDERWATER MINING INSTITUTE (16th Annual), Halifax, Nova Scotia, Canada. (General Secretary, U.M.I. ${ }^{85}$, e/o Ocean Mining Division, Canada Oil and Gas Lands Administration, 355 River Road, Ottawa, Ontario, Canada K IL 8Cl)

October $21-25,1985$

- ARID LANDS: TODAY AND TOMORROW (International Conference), Tueson, Arizona, U.S.A. (G.P. Nabham, Office of Arid Land Studies, University of Arizona, Tucson, AZ 85721, U.S.A.)

October $28-31,1985$

- GEOLOGICAL SOCIETY OF AMERICA (Annual
Meeting) Orlando, Fla., U.S.A. (S.S. Beggs, Geological Society of America, P.O. Box 91 40, 330 Penrose Place, Boulder, CO 80301 , U.S.A.)

October 28 - November 1,1985

COAL SCIENCE (International Conference) Sydney, Australia. Sponsored by JEA (R.W. Hinde, CSIRO Div. of Fossil Fuels, Box 136, North Ryde, NSW 2113, Australia)

October 29 - November 2,1985

INDONESIAN MINING (International Exhibition), Jakarta, Indonesia. (Overseas Exhibition Services, 11 Manchester Square, London WIM 5 AB, U.K.)

November 1985

- PETROLEUM GEOCHEMISTRY AND EXPLORATION IN AFRO-ASIAN REGION (International Conference), Dehradun, India. (Kuldeep Chandra, KDM Institute of Petroleum Exploration, Oil and Natural Gas Commission, 9, Kaulagarh Road, Dehradun - 248195 , India)

November 1985

DESERTIFICATION (International Symposium), Khartoum, Sudan. (Dr. W.G. Sombroek, ISSS, International Soil Museum, 9 Duivendaal, POB 353,6700 A.J. Wageningen, The Netherlands)

December $9-13,1985$

AMERICAN GEOPHYSICAL UNION (Fall Meeting), San Franciseo, California, U.S.A. (AGU Meetings 2000 Florida Avenue NW, Washington, D.C. 20009 , U.S.A.)

\section{6}

1986

CIRCUM-PACIFIC ENERGY MINERALS (Meeting), Singapore. (American Association of Petroleum Geologists, Box 979, Tulsa, OK 74101, U.S.A.)

1986

\ CARBONIFEROUS STRATIGRAPHY (IUGS Subcommission Field and General Meeting), Argentina. (Dr. W.B. Saunders, Secretary SCCS, Department of Geology, Bryn Mawr College, Bryn Mawr, PA 19010, U.S.A.)

February 1 - 9, 1986

INTERNATIONAL VOLCANOLOGICAL CONGRESS, Auckland, Hamilton, Rotorua, New Zealand. Sponsored by IAVCEI. (P.E. Baker, Department of Earth Sciences, University of Leeds, Leeds LS2 9JT, U.K.)

April $21-24,1986$

- ENVIRONMENTAL GEOTECHNOLOGY (Interna tional Symposium), Allentown, Penn., U.S.A. (Prof. H.Y. Fang, Symposium Chairman, Geotechnical Engineering Division, Department of Civil Engineering, Lehigh University 13 , Bethlehem, PA 18015 , U.S.A.)

April $24-27,1986$

INDUSTRIAL MINERALS (7th International Congress), Athens, Greece. (G.M. Clarke, Editor, Industrial Minerals, 16 Lower Marsh, London SEl, U.K.)

May 11-16, 1986

MINING AND METALLURGICAL INSTITUTIONS (13thCongress), Canberra, Australia. (Council of Mining and Metallurgical Institutions, c/o The Australian Institute of Mining and Metallurgy, P.O. Box 310 , Carlton South, Vietoria, Australia 3053)

May $19-21,1986$

GEOLOGICAL, MINERALOGICAL ASSOCIATIONS OF CANADA (Joint Annual Meeting), Ottawa, Ontario, Canada. (Dr. J.A. Donaldson, Department of Geology, Carleton University, Ottawa, Ontario, Canada K IS 5B6)

May $19-23,1986$

- american Geophysical union (Spring Meeting), Baltimore, Maryland, U.S.A. (AGU Meetings, 2000 Florida Avenue NW, Washington, DC 20009, U.S.A.)
June 1 - 5, 1986

GEOSCIENCE INFORMATION (3rd International Conference) Adelaide, South Australia. (Conference Secretariat $31 \mathrm{CGl}$, c/o Australian Mineral Foundation, Private Bag 97, Glenside, South Australia, 5065, Australia)

June 2 - 5, 1986

DINOSAUR SYSTEMATICS (Symposium) Drumheller, Alberta, Canada. (Kenneth Carpenter, Academy of Natural Seiences, 19th and the Parkway, Philadelphia, PA 19103, U.S.A.)

June $3-6,1986$

EUROPEAN ASSOCIATION OF EXPLORATION GEOPHYSICISTS (Meeting), Ostend, Belgium. (E. van der Gaag, European Association of Exploration Geophysicists, P.O. Box 162, NL-2501 AN The Hague, The Netherlands)

June $15-18,1986$

- AMERICAN ASSOCIATION OF PETRULEUM GEOLOGISTS (Annual Convention), Atlanta, Georgia, U.S.A. (Howard Cramer, Emory University, Department of Geology, Atlanta, GA 30322 , U.S.A.)

Summer 1986

GEOCHRONOLOGY, COSMOCHRONOLOGY AND ISOTOPE GEOLOGY (6th International Conference), Cambridge, U.K. Sponsored by IAVCEI, (P.E. Baker, Department of Earth Sciences, University of Leeds, Leeds LS2 9JT, U.K.)

July 1986

INTERNATIONAL ASSOCIATION OF HYDROLOGICAL SCIENCES (2nd General Assembly), Budapest, Hungary. (Dr. A. Szöllosi-Nagy, VITUKI, HI-1 453, Budapest, Pf 27, Hungary)

July $13-18,1986$

* INTERNATIONAL MINERALOGICAL ASSOCIATION (General Meeting), Stanford, Calif., U.S.A. (Prof. C.T. Prewitt, Department of Earth and Space Sciences, State University of New York, Stony Brook, NY 11794 , U.S.A.)

July ] $4-18,1986$

PRECAMBRIAN GOLD/URANIUM DEPOSITS (GSSA Congress), Johannesburg, South Africa. (C.F. Vermaak, Geological Society of South Africa, Kelvin House, 2 Holland Street, Johannesburg 2001, South Africa)

August $11-15,1986$

KIMBERLITE (4th International Conference), Perth, Western Australia. (Dr. A.F. Trendall, Geological Survey of Western Australia, 66 Adelaide Terrace, Perth, W.A., Australia)

A ugust $13-20,1986$

SOIL SCIENCE (13th ISSS International Congress), Hamburg, F.R.G. (Prof. Dr. K.H. Hartge, Inst, für Bodenkunde, Univ. Hannover, Herren-häserstrasse 2, D-3000 Hannover 21, F.R.G.)

August $17-22,1986$

CIRCUM-PACIFIC ENERGY AND MINERAL RESOURCES (Conference), Singrpore. (A.G. Hatley, Graffney Chine and Associates, Inc., P.O. Box 796309, Dallas, TX 75379 , U.S.A.)

A ugust $18-22,1986$

* INTERNATIONAL ASSOCIATION ON THE GENESIS OF ORE DEPOSITS (7th Symposium), Lulea, Sweden. (Centek Conference, S-951 87 Lulea, Sweden)

August $24-30,1986$

* INTERNATIONAL ASSOCiation OF SEDIMENTOLOGISTS (12th International Congress), Canberra, Australia. (ACTS, GPO Box 1929, Canberra ACT 2601, Australia)

\section{September 7 - 12, 1986}

REMOTE SENSING IN GLACIOLOGY (Symposium), Cambridge, England. (Secretary General, International Glaciological Society, Lensfield Road, Cambridge CB2 lER, England, U.K.)

September 8 - 12, 1986

PALEOCEANOGRAPHY (2nd International Con- 
ference), Woods Hole, U.S.A. (W.A. Berggren, Woods Hole Oceanographie Institute, Woods Hole, MA 02543, U.S.A.)

Oetober 5 - 11, 1986

WORLD ENERGY (13th Congress), Cannes,

France. (R. Ruttley, World Energy Conference, 34 St. James' Street, London SWIA IHD, U.K.)

October $20-26,1986$

* INTERNATIONAL ASSOCIATION OF ENGINEERING GEOLOGY (5th Congress), Buenos Aires, Argentina. (Dr. M. Primel, L.C.P.C., 58, boulevard Lefebvre, 75732 Paris Cedex 15, France)

November $2-6,1986$

SOCIETY OP EXPLORATION GEOPHYSICISTS (56th Annual Meeting), Houston, Texas, U.S.A. (Convention Assistant, Soeiety of Exploration Geophysicists, P.O. Box 3098, Tulsa, OK 74101 , U.S.A.

November $10-13,1986$

GEOLOGICAL SOCIETY OF AMERICA (AnnUA) Meeting), San Antonio, Texas, U.S.A. (S.S. Beggs, Geological Socicty of America, P.O. Box 9140,3300 Penrose Place, Boulder, CO 80301. U.S.A.)

\section{7}

May $25-27,1987$ GEOLOGICAL,
TIONS OF CANADA (Joint Annual Meeting), Saskatoon, Canadn. (Dr. W.O. Kupseh, Department of Geologieal Seiences, University of Sarkatchewan, Saskatoon, Seskatehewan, Canade S7N 0W0)

June 1987

- AUSTRALIA'S INTERNATIONAL MINING AND EXPLORATION RXHIBTTON 87 (Meeting), Sydney, Australia. (Thomson Bxhibitions, 47 Chippen Street, Chippendale, NSW 2008, Australia)

June $7-10,1987$

- AAPG and SEPM (Annual Meeting), Los Angeles, Calif., U.S.A. (AAPG Headquarters, Box 979, Tulsa, OK 74101, U.S.A.

July 31 - August 9,1987

* INTERnational union for Quaternary RESEARCH (12th Congress), Ottawa, Ontario, Canada. (Dr. Alan V. Morgan, Department of Earth Seienees, University of Waterloo, Waterloo, Ontario, Canada N2L 3GI)

August $9-22,1987$

IUGG (XIX General Assembly), Vaneouver. Canada (R.D. Russell, Dept. Geophysies and Astronomy, University of British Columbia, Vancouver, B.C., Canade v6T IW5)

September 1987

INTERNATIONAL SOCIETY FOR ROCK MECHANICS (6th International Congress), Montreal,
Canadn. (Prof. B. Ladanyi, Dept. Civit Engineering, Ecole Polytechnique, Box 6079, Stn. A, Montréal, Canada H3C 3A7)

September $7-11,198$ ?

- CARBONIFEROUS STRATIGRAPHY AND GEOLOGY (11th International Congress), Beijing. People's Republie of China. (Prof. Yang Jing-zhi, Nanjing Institute of Geology and Palaeontology, Chi-Ming-Ssu, Nanjing, People's Republic of China)

Oetober $11-15,1987$

- SOCIETY OF EXPLORATION GEOPHYSICISTS (Annual Meeting), New Orleans, L.a., U.S.A. (Marvin R. Hewitt, Amoco Production Co., Box 591, Tulsa, OK 74102, U.S.A.)

October $26-29,198$ ?

- GEOLOGICAL SOCIETY OF AMERICA (Annual Meeting), Phoenix, Arizonn, U.S.A. (Jean M. Latulippe, GSA Headquarters, Box 9140 , Boulder, CO 80301, U.S.A.)

Wish to announce meetings of international signifieance? Send brochure or details to EPISODES, Room 177, 601 Booth Street, Ottawa, Canada KIA OE8, at least one month in advance of publiention dete.

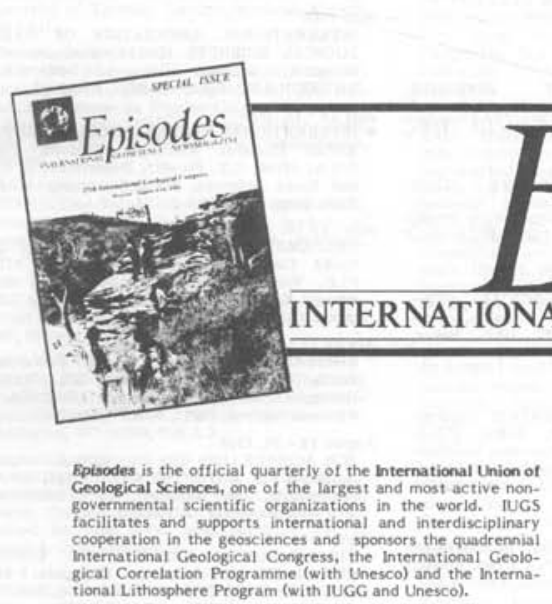

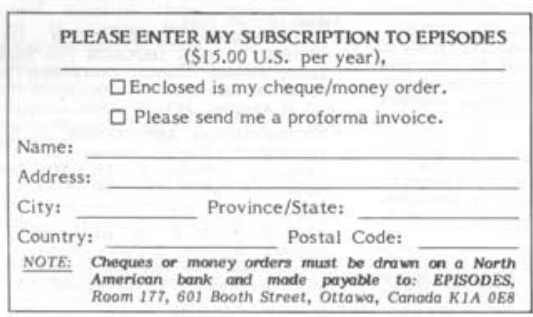

Earth scientists must contend with rapidly advancing knowledge and an ever-growing information base. They must also keep pace with a bewildering diversity of organizations and a wide array of conferences, seminars and workshops. Episodes helps solve these problems by covering both scientific and organizational news. Feature articles - contributed by leading geoscientists from all parts of the world - review developments at the frontiers of the earth sciences.

$$
\begin{aligned}
& \text { Each issue carries: } \\
& \text { - State-of-the-Art Reviews } \\
& \text { - Conference Reports } \\
& \text { - News ftems } \\
& \text { - Book Reviews } \\
& \text { - Calendar of Internotional } \\
& \text { Events } \\
& \text { - New Books and Maps }
\end{aligned}
$$

With these features, Episodes is an essential information source and planning guide for active scientists. We urge you to subscribe to this leading international geoscience newsmagazine.

\section{Your Window to the World}

